\title{
Pseudodicentric chromosome 18 diagnosed by chromosome painting and primed in situ labelling (PRINS)
}

\author{
C A Brandt, B Djernes, H Strømkjær, M B Petersen, S Pedersen, J Hindkjær, \\ J Brinch-Iversen, G Bruun-Petersen
}

\begin{abstract}
We report on a newborn white male infant with marked dysmorphic features and various congenital malformations. The initial clinical evaluation showed Crouzon-like features as well as some features of trisomy 18 syndrome and trisomy 13 syndrome. The results from conventional cytogenetic analysis showed a structurally abnormal chromosome replacing one normal chromosome 18 , but only by applying molecular cytogenetic methods could the architecture of this abnormal chromosome be characterised clearly. The primed in situ labelling (PRINS) technique, using a newly synthesised $\propto 18$ oligonucleotide, showed the dicentric pattern and direct chromosome painting established the origin to be from chromosome 18. The combination of conventional cytogenetics and molecular cytogenetics showed the karyotype in the proband to be $45, X Y,-14,-18,-21$, $+t(14 ; 21)$, + psu dic(18) (qter $\rightarrow$ cen $\rightarrow$ p11.3: :p11.3 $\rightarrow$ psu cen $\rightarrow$ qter). This was supported by molecular analysis using chromosome 18 specific DNA markers, which showed the paternal origin of the abnormal chromosome.
\end{abstract}

(f Med Genet 1994;31:99-102)

Isochromosome 18 is an uncommon cytogenetic aberration in newborn infants; only about 26 cases have been reported with $i(18 p)$ and 16 cases with $i(18 q) .{ }^{1}$ All cases of $i(18 p)$ are supernumerary in contrast to $\mathrm{i}(\mathbf{1 8 q})$. Isodicentric chromosomes of both short and long arms replacing one normal chromosome 18 are even more rarely reported, ${ }^{1}$ because the dicentric pattern is difficult to establish in all cases using conventional banding analysis alone.

We have used direct chromosome painting, the primed in situ labelling (PRINS) technique, and DNA analysis to characterise a constitutional pseudodicentric chromosome 18 , psu dic(18), found in a growth retarded male infant with marked dysmorphic features and various congenital malformations.

\section{Case report}

The proband was the first child of healthy, non-consanguineous white parents, both 26 years of age. There was no family history of genetic diseases. Spontaneous labour started at term and there were 2.81 of clear amniotic fluid. The child had an Apgar score of 0 at birth and resuscitation was started immediately. Scores of 1,3 , and 6 were recorded at one, five, and 10 minutes, respectively. He was hypotonic and had symptoms of respiratory distress.

Physical examination showed a mature, but growth retarded, male infant with marked dysmorphic features (fig 1). Birth weight was $2600 \mathrm{~g}$, head circumference $29.5 \mathrm{~cm}$ (below the 3 rd centile), crown-rump length $35 \mathrm{~cm}$, and crown-heel length $49 \mathrm{~cm}$. He was microcephalic and brachycephalic with ptosis and proptosis of both eyes but without hypertelorism. There was midface hypoplasia with the maxillary complex being mildly reduced sagittally, but there was symmetrical prominence of the zygoma. The nose was slightly deviated but firm and a marked swelling of the mucosal membranes was causing almost complete occlusion of the passage. A long and flat philtrum was apparent. The ears were low set and large but of normal shape. The lower jaw was normal. A lateral mucosal swelling of the palate was noted, causing a high, arched palate. The hands were ulnarly deviated to a mild degree with contracture of all proximal interphalangeal joints and overlapping of the second and fifth fingers. Simian creases were not present. The feet were short, broad, and in a slight valgus position, with broad big toes and mild "sandal gap". Rocker bottom feet were not present. A systolic cardiac murmur was heard.

A CT scan showed almost complete closure of the coronal suture with only narrow openings in the lowest areas. The remaining sutures, especially the lambdoid suture, were markedly reduced in width.

He developed marked conjugated hyperbilirubinaemia (total serum bilirubin $340 \mathrm{mmol} / 1$ with 125 conjugated). At 10 days of age both conjugated and unconjugated serum bilirubin suddenly decreased spontaneously, but increas- 


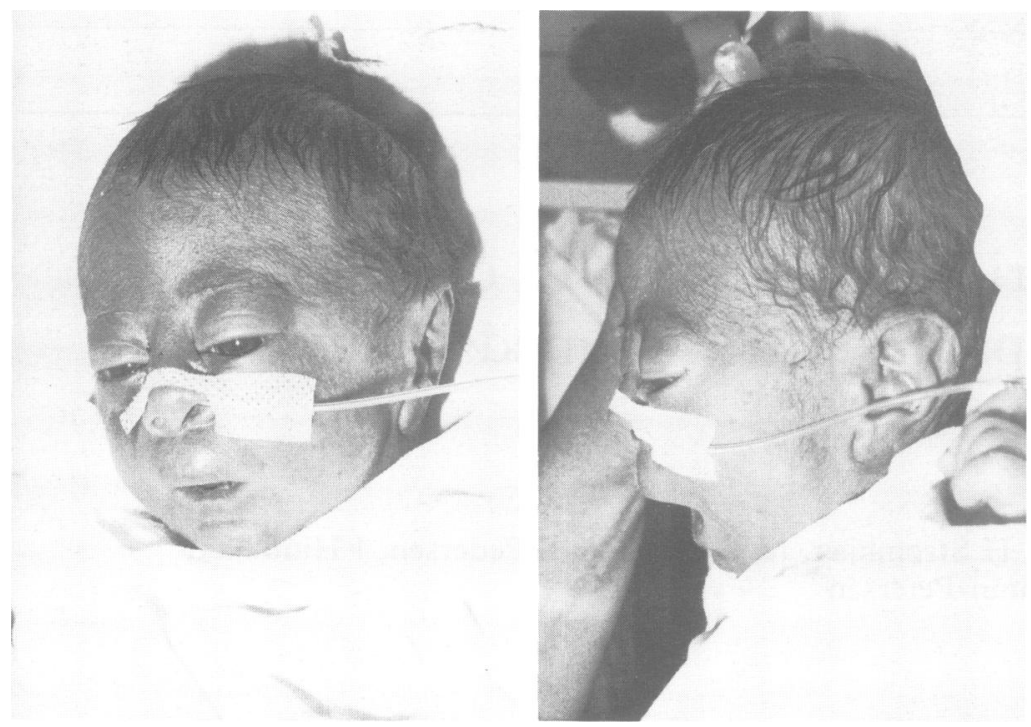

Figure 1 The proband at 1 week of age. Note the dysmorphic features and the low set ear.

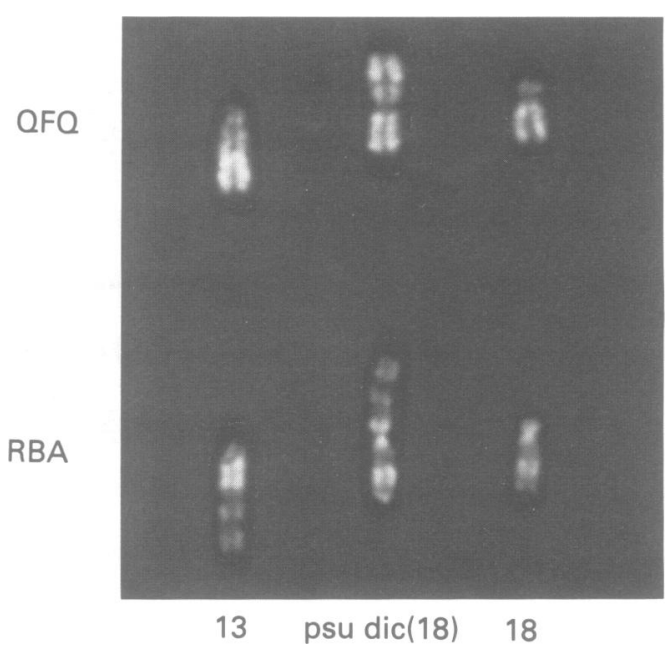

Figure 2 The structurally abnormal chromosome 18 is shown here between the normal chromosomes 13 and 18. The longest arm can easily be mistaken for the distal part of the long arm of chromosome 13.

distal part of the short arm of chromosome 18 and partially trisomic for the distal part of the long arm of chromosome 13. Only one centromere was apparent on the structurally abnormal chromosome. range $308 \mathrm{~g} \pm 76 \mathrm{~g}$ ). The hemispheres were symmetrical with almost complete absence of the temporal poles. There was, however, partial development of the Sylvian sulcus. Gyri and sulci were symmetrical. The cerebellum and the chiasma opticum were macroscopically normal. The third ventricle was dilated and was communicating with the frontal and lateral ventricles. The septum pellucidum was absent, but the corpus callosum was normal. Sequelae of previous haemorrhage were seen in the walls of the fourth ventricle. Examination of the heart showed a VSD and only two valves in the aortic and pulmonary ostia. No other visceral abnormalities were observed.

\section{Methods and results}

CONVENTIONAL CYTOGENETICS

Chromosome studies were performed on cultures from a skin biopsy and lymphocytes of the proband, and on lymphocyte cultures from the parents. Metaphases were stained with quinacrine mustard (QFQ banding) according to standard techniques. Analysis showed an unbalanced karyotype $45, \mathrm{XY},-14,-18,-21$, $+t(14 ; 21),+t(18 ; ?)$. Efforts were made to identify the origin of the long arm of the obviously asymmetrical chromosome by examining prometaphases using high resolution RBA banding. The karyotype of the proband's mother was $46, \mathrm{XX}$ and the father's karyotype was $45, \mathrm{XY}, \mathrm{t}(14 ; 21)$. All 50 metaphases analysed from the proband showed 45 chromosomes including the $t(14 ; 21)$ from the father (not shown) and the abnormal chromosome (fig 2). The abnormal chromosome was composed of the long arm of a chromosome 18 and an even longer arm, which was thought to be composed of a deleted short arm of chromosome 18 and, judging by the RBA examination, the distal part of the long arm of chromosome 13. Therefore, the patient was initially believed to be partially monosomic for the

\section{CHROMOSOME PAINTING} enting chromosomes 3,13, and 18 (chosen because of the results obtained by conventional cytogenetics) were generated from flow sorted normal chromosomes, amplified, and labelled with biotin-11-dUTP using a degenerate oligonucleotide primed polymerase chain reaction (DOP-PCR), as described by Pedersen et al. ${ }^{2}$ The chromosome paintings were accomplished essentially as described by Pinkel $e t a l^{3}$ using the chromosome in situ suppression (CISS) hybridisation technique. The hybridised DNA was detected with FITC-avidin. Painting with DNA libraries representing chromosomes 3 and 13 showed total painting of two sets of normal chromosomes 3 (not shown) and 13 (fig 3a), whereas painting with a DNA library specific for chromosome 18 resulted in one normal chromosome 18 and total painting of the structurally abnormal chromosome (fig $3 \mathrm{~b}$ ). The abnormal chromosome was therefore established to be composed exclusively of chromosome 18 material.

\section{PRIMED IN SITU LABELLING (PRINS)}

PRINS was performed essentially as described by Hindkjær et al. ${ }^{4}$ An oligonucleotide $(\alpha 18$, 43 nucleotides, Koch et al, unpublished data, but available on request) representing sequences specific for the chromosome 18 centromere was used as primer in a chain elongation with digoxigenin-11-dUTP as the labelled nucleotide. The incorporated labelled nucleotide was detected with anti-digoxigenin-fluorescein Fab fragments (Boehringer Mannheim). The result showed two centromeres, one active and one inactive, in the abnormal chromosome, indicating a dicentric chromosome originating
Chromosome specific DNA libraries repres- 

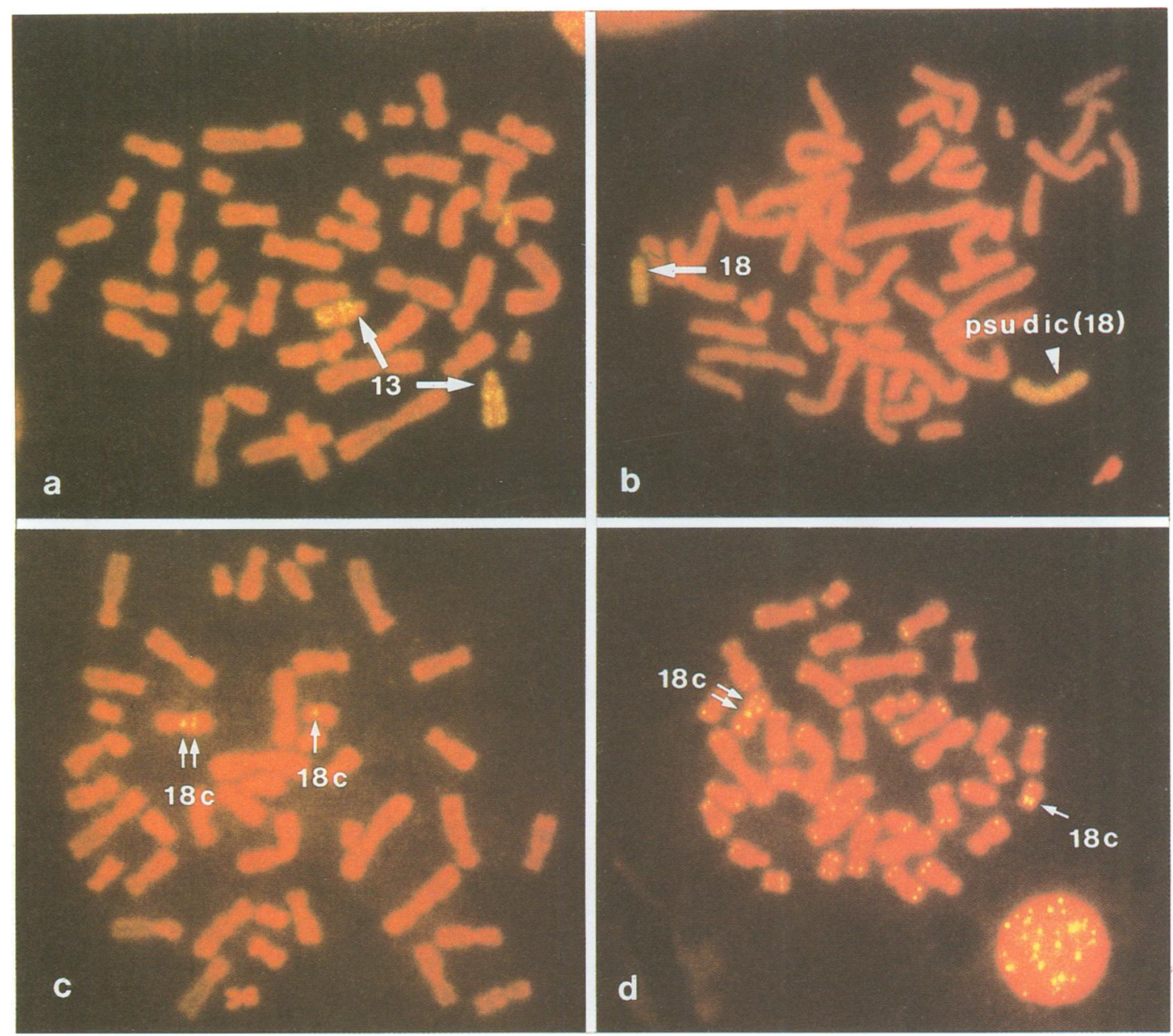

Figure 3 Chromosome painting with DNA libraries specific for chromosomes 13 and 18 shows two normal chromosomes 13 (a), but only one normal 18 together with total painting of the abnormal chromosome 18 (b). Primed in situ labelling (PRINS) with the $\alpha 18$ oligonucleotide shows two centromeres on the psu dic (18), one active and one inactive (two closely bound signals) (c). PRINS with telo 2 oligonucleotide shows normal telomeres on the normal chromosome 18 and on the psu dic (18) (d).

from chromosome 18 (fig 3c). The same centromere was inactive in all 70 metaphases and prometaphases analysed on re-examination of the cytogenetic preparations.

Another PRINS reaction, using two different oligonucleotides simultaneously, was performed with a $42 \mathrm{mer}$ oligonucleotide $\left((\mathrm{CCCTAA})_{7}\right.$, telo 2$)$ as a primer to visualise the telomeres, along with the $\alpha 18$ oligonucleotide described above. The telo 2 oligonucleotide is a 7 mer of a hexanucleotide consensus sequence representing the human telomeres (TTAGGG), identified by Moyzis et al..$^{5}$ The results showed that the aberrant chromosome 18 had only telomeric sequences at the chromosome ends (fig $3 \mathrm{~d}$ ), so the breakpoint has to be subtelomeric for both short arms in the abnormal chromosome 18. The centromere specific primer was used to identify the abnormal chromosome in propidium-iodide stained preparations (no banding pattern) and to define the region within which the fusion point must be located.

MOLECULAR ANALYSES

DNA was extracted from a skin fibroblast culture from the proband and from lymphoblastoid cell lines of the parents. DNA polymorphisms resulting from microsatellites were studied after PCR amplification ${ }^{6}$ using end labelling of primers, polyacrylamide gel electrophoresis, and autoradiography. ${ }^{7}$ The microsatellites studied were: (1) a (CA), repeat at the D18S40 locus (located at 18p11.21-pter), ${ }^{8}(2)$ a $(\mathrm{CA})_{\mathrm{n}}$ repeat at the D18S41 locus (located at $18 \mathrm{q} 21.2-\mathrm{q} 21.3),{ }^{8}$ (3) a $(\mathrm{CA})_{\mathrm{n}}$ repeat at the D18S50 locus (located at 18q23-qter), ${ }^{8}$ and (4) a (ATGG) and (TGGA) repeat at the MBP gene (located at 18q23-qter). ${ }^{89}$ The scoring of genotypes was as previously described by Petersen et al. ${ }^{10}$ The results are shown in the table. By dosage comparison of alleles, the markers studied showed a duplication of a paternal allele.

\section{Discussion}

In the present case, initial clinical evaluation of the proband showed Crouzon-like features including craniosynostosis, maxillary hypo-

DNA polymorphism analysis in a case of pseudodicentric chromosome 18

\begin{tabular}{llll}
\hline & \multicolumn{2}{l}{ Genotypes* } & \\
\cline { 2 - 4 } Locus & Father & Proband & Mother \\
\hline D18S40 & 13 & 233 & 24 \\
D18S41 & 23 & 224 & 14 \\
D18S50 & 12 & 113 & 13 \\
MBP & 23 & 223 & 13 \\
\hline
\end{tabular}

* The numbers represent the different alleles at a specific locus. 
plasia, shallow orbits, and ocular proptosis. Marked dysmorphic features and other congenital malformations could not, however, be explained by this autosomal dominantly inherited disease, so chromosome studies were initiated. Partial trisomy $13 \mathrm{q}$ was suggested, based on conventional cytogenetic analysis alone, but this did not agree with the clinical features. Further cytogenetic investigations were therefore performed using molecular cytogenetic methods. The PRINS technique $^{1112}$ turned out to be an easy, fast, and sensitive method of establishing the dicentric pattern of the structurally abnormal chromosome using a newly synthesised $\alpha 18$ oligonucleotide as the primer. Direct chromosome painting established unequivocally the origin of the abnormal chromosome to be exclusively from chromosome 18, because of the total painting with a highly chromosome 18 specific DNA library. The karyotype of the proband was therefore $45, \mathrm{XY},-14,-18,-21,+\mathrm{t}(14 ; 21)$, + psu $\operatorname{dic}(18)($ qter $\rightarrow$ cen $\rightarrow$ p1 1.3: : p1 1.3 $\rightarrow$ psu cen $\rightarrow$ qter) with the $14 ; 21$ translocation of paternal origin. The proband was partially monosomic for the short arm and trisomic for the long arm of chromosome 18. DNA polymorphism analysis showed the abnormal chromosome to be of paternal origin, and an association between the $14 ; 21$ translocation and a higher susceptibility to chromosomal breakage in the father cannot be excluded.

There is considerable variability in the phenotype of the $18 \mathrm{p}$ - syndrome ${ }^{13}$ and the features of $18 \mathrm{p}$ - are normally present in all cases of $\mathrm{i}(18 \mathrm{q}) .{ }^{114}$ Cases with idic(18) have more or less pronounced features of $18 \mathrm{p}$ - depending on the size of the deletion in the short arm of chromosome 18 or the severity of trisomy 18 symptoms. ${ }^{15}$ Symptoms of monosomy $18 \mathrm{p}$ may be masked by the more severe clinical manifestations of trisomy $18 \mathrm{q}$ as suggested by FrosterIskenius et al. ${ }^{16}$ In our case, the findings of microcephaly, ptosis, and large ears may be explained by a deletion of chromosomal material in $18 \mathrm{p}$. The breakpoint was thought to be in band p11.3, based mainly on cytogenetic analysis, and was supported by the result obtained using the DNA marker D18S40, which has been mapped to p11.21-pter. The findings of low set ears, overlapping fingers, and congenital heart disease were features compatible with trisomy 18 which were supported by the diagnosis of the pseudodicentric chromosome 18. However, the proband lacked the malformations of the internal organs characteristic of trisomy $18,{ }^{15}$ except for the heart malformation. This might be explained by a position effect variegation which describes the variable expression of a euchromatic gene which can arise from chromosomal rearrangements, insertions, deletions, or local regions of inactivation. This could be the case with iso- chromosomes and especially in this case with marked morphological alterations in the long arm of the asymmetrical chromosome. We did not detect any deletion using DNA markers on chromosome 18, but an inversion or other rearrangements in $18 \mathrm{q}$ are possible.

Using molecular cytogenetic methods to supplement standard banding analysis proved to be a good strategy for identifying the chromosomal aberration in this particular case. We conclude that in the characterisation of structural chromosome abnormalities, the use of modern molecular cytogenetic methods is a valuable tool in obtaining the most precise analysis of the karyotype.

We would like to acknowledge Maja Kristensen for skilful technical assistance. Svend-Erik Madsen, Soren Haslov, and Kirsten Millgard are thanked for the photographic work. We thank Jorn Koch and A J Therkelsen for providing ws with the thank Jørn Koch and A Therkelsen for providing us with the oligonucleotide $\alpha 18$ and telo 2 , respectively. Tracey Flint is thanked for help with language and Dr Steen Kolvraa for his useful comments and editing of the article. This work wa (the Danish Human the Danish Cancer Society (JH).

1 Meguid NA, Habibian R. Isodicentric chromosome 18 in an Meguid NA, Habibian R. Isodicentric chromosome 18 in an abnormal infant using chrom

2 Pedersen S, Hindkjær J, Brandt CA, Bolund L, Kølvraa S. Reverse chromosome painting. In: Choo KHA, ed. Methods in molecular biology. In situ hybridization protocols. New Jersey: Humana Press (in press)

3 Pinkel D, Landegent J, Collins C, et al. Fluorescence in situ hybridization with human chromosome-specific libraries: detection of trisomy 21 and translocation of chromosome 4. Proc Natl Acad Sci USA 1988;85:9138-42.

4 Hindkjær J, Koch J, Mogensen J, et al. Primed in situ abelling of nucleic acids. Int $\mathcal{f}$ Biotech 1991;12:752-6.

5 Moyzis RK, Buckingham JM, Cram LS, et al. A highly conserved repetitive DNA sequence, (TTAGGG), present at the telomeres of human chromosomes. Proc Natl Acad Sci USA 1988;85:6622-6.

6 Saiki RK, Scharf S, Faloona F, et al. Enzymatic amplification of beta-globin genomic sequences and restriction site analysis for diagnosis of sickle cell anemia. Science 1985;230:1350-4.

7 Petersen MB, Frantzen M, Antonarakis SE, et al. Comparative study of microsatellite and cytogenetic markers for in Down syndrome. Am $\mathcal{F}$ Hum Genet 1992;51:516-25.

8 Straub RE, Speer MC, Luo Y, et al. A microsatellite genetic linkage map of human chromosome 18. Genomics linkage map

9 Polymeropoulos $\mathrm{MH}$, Xiao H, Merril CR. Tetranucleotide repeat polymorphism at the human myelin basic protein repeat polymorphism at the human myelin

10 Petersen MB, Schinzel AA, Binkert F, et al. Use of short sequence repeat DNA polymorphisms after PCR amplification to detect the parental origin of the additional chromosome 21 in Down syndrome. Am 7 Hum Genet 1991;48:65-71.

11 Koch JE, Kølvraa S, Petersen KB, Gregersen N, Bolund L. Oligonucleotide-priming methods for the chromosomespecific labelling of alpha satellite DNA in situ. Chromosoma 1989;98:259-65.

12 Koch J, Hindkjær J, Mogensen J, Kølvraa S, Bolund L. An mproved method for chromosome-specific labelling of alpha satellite DNA using denatured doublestranded DNA probes as primers in a PRimed IN Situ labelling (PRINS). Genet Anal Tech Appl 1991;8:171-8.

13 Smith DW. Chromosomal abnormality syndromes. In: Jones KL, ed. Smith's recognizable pattern of human malformation. Philadelphia: Saunders, 1988:10-74.

14 Bass HN, Sparkes RS, Miller AA. Features of trisomy 18 and $18 \mathrm{p}$ - syndromes in an infant with $46, \mathrm{XY}, \mathrm{i}(18 \mathrm{q})$. Clin Genet 1979;16:163-8.

15 Fioretti G, Stabile M, Pagano L, et al. A case of Edward's syndrome with pseudodicentric isochromosome 18: 46,XY, $\operatorname{dic}(18)$ (p11: p11). Ann Genet (Paris) $1982 ; 25: 116-18$

16 Froster-Iskenius U, Coerdt W, Rehder H, Schwinger E. Isochromosome $18 \mathrm{q}$ with karyotype $46, \mathrm{XX}, \mathrm{i}(18 \mathrm{q})$. Cytogenetics and pathology. Clin Genet 1984;26:549-54. 